Check for updates

Cite this: RSC Adv., 2018, 8, 39650

\title{
Allotropes of tellurium from first-principles crystal structure prediction calculations under pressure
}

\author{
Yuan Liu, (D) $\dagger^{\mathrm{ab}}$ Shunbo Hu, (D) $\dagger^{\mathrm{a}}$ Riccarda Caputo, (D) ${ }^{\mathrm{c}}$ Kaitong Sun, ${ }^{\text {ab }}$ Yongchang $\mathrm{Li}^{\mathrm{ab}}$ \\ Guodong Zhao (D) ab and Wei Ren (D)*ab
}

We investigated the allotropes of tellurium under hydrostatic pressure based on density functional theory calculations and crystal structure prediction methodology. Our calculated enthalpy-pressure and energy-volume curves unveil the transition sequence from the trigonal semiconducting phase, represented by the space group $P 3_{1} 21$ in the range of $0-6 \mathrm{GPa}$, to the body centered cubic structure, space group $\operatorname{Im} \overline{3} \mathrm{~m}$, stable at $28 \mathrm{GPa}$. In between, the calculations suggest a monoclinic structure, represented by the space group $C 2 / m$ and stable at $6 \mathrm{GPa}$, and the $\beta$-Po type structure, space group $R \overline{3} \mathrm{~m}$, stable at $10 \mathrm{GPa}$. The face-centered structure is found at pressure as high as $200 \mathrm{GPa}$. As the pressure is increased, the transition from the semiconducting phase to metallic phases is observed.

Received 21st September 2018 Accepted 19th November 2018

DOI: $10.1039 / c 8 r a 07843 b$

rsc.li/rsc-advances

11.5 GPa is of the $\beta$-Po type (space group $R \overline{3} m) .{ }^{18}$ Therefore, the

\section{Introduction}

Tellurium is a semiconductor with a high thermoelectric figure of merit comparable to $\mathrm{GeSi}^{1}{ }^{1} \mathrm{BiSb}^{2}$ or other well-known materials. $^{3-5}$ The chiral tellurium has been investigated for its anisotropic lattice thermal conductivity and p-type transport properties from anisotropic hole pockets of the Fermi surface. ${ }^{6,7}$ In addition, very recently, structures with few-layers of tellurium were studied as two-dimensional topological materials. ${ }^{8,9}$ In fact, it presents topological properties for electronic transport, benefitting from the nonsymmorphic screw symmetry, the lonepair electrons and the strong spin-orbit interaction. ${ }^{10-12}$ For the ground-state chiral tellurium, the band splitting and band inversion were found upon the application of shear strain or pressure $^{13-15}$ together with the existence of topological Weyl nodes near the Fermi level. ${ }^{13,14}$

Regarding the crystal structure, tellurium undergoes several phase transitions under pressure. Though there is a lack of consensus of the exact phase transitions, the sequence reported in the literature suggests the transition from the ground state, represented in the trigonal space group $P 3_{1} 21$ to a puckered layer phase at $4 \mathrm{GPa},{ }^{16}$ and then to the monoclinic space group $P 2_{1}$ and to an orthorhombic structure in the pressure range of 6.6-10 GPa. ${ }^{16,17}$ Powder X-ray diffraction (XRD) measurements at $5.3 \mathrm{GPa}$ and $11.5 \mathrm{GPa}$ led to the conclusion that the structure at

${ }^{a}$ Materials Genome Institute, International Centre for Quantum and Molecular Structures, and Department of Physics, Shanghai University, Shanghai 200444, China.E-mail: renwei@shu.edu.cn

${ }^{b}$ Shanghai Key Laboratory of High Temperature Superconductors, Shanghai University, Shanghai 200444, China

${ }^{c}$ International Centre for Quantum and Molecular Structures, Shanghai University, Shanghai 200444, China

$\dagger$ These authors contributed equally to this work. overall phase transitions are from the trigonal, space group $P 3_{1} 21$ (Te-I) to the monoclinic, space group $P 2_{1}$ (Te-II) at about $4 \mathrm{GPa} ;{ }^{16}$ from Te-II to the orthorhombic phase (Te-III) at about $7 \mathrm{GPa}$; from Te-III to the rhombohedral phase in the trigonal space group $R \overline{3} m$ (Te-IV) at about $10.5 \mathrm{GPa}$; from Te-IV to the body-centered cubic phase (Te-) at $27 \mathrm{GPa} .^{18,19}$ To the best of our knowledge, the symmetry representation of the orthorhombic phase is not reported in the literature. In contrast to that, the Xray structural analysis ${ }^{20}$ ruled out the second-order phase transition from the monoclinic $P 2_{1}$ to the orthorhombic phase and then to the trigonal $R \overline{3} \mathrm{~m}$, and suggested that the monoclinic should be represented instead in the $C 2 / m$ space group. ${ }^{20}$ Therein the authors ${ }^{20}$ suggested the phase transitions as increasing the pressure as follows: trigonal $\rightarrow$ puckered monoclinic $\rightarrow$ monoclinic $\rightarrow$ rhombohedral $\rightarrow$ body-centered cubic (bcc). Hejny and McMahon ${ }^{21,22}$ further showed that Te-II was a triclinic structure and Te-III was an incommensurate monoclinic structure. Accordingly, the phase transition sequence they proposed is from the trigonal phase, consisting of helical spiral chains, to the commensurate triclinic structure, comprising puckered layers that contain zig-zag chains with alternating long and short bonds (such values are similar to SeIII $C 2 / m){ }^{21}$ to the incommensurate monoclinic structure with a superspace group $I^{\prime} 2 / m(0 \mathrm{q} 0) \mathrm{s} 0$ (with $a=3.288 \AA, b=4.010 \AA, c$ $\left.=2.589 \AA, \beta=112.98^{\circ}\right),{ }^{22}$ and finally to a bcc phase. Therefore, the phase transition sequence investigated by Hejny et al. is as follows: trigonal $\rightarrow$ triclinic $\rightarrow$ monoclinic $(\rightarrow \beta$-Po rhombohedral under high pressure and high temperature) $\rightarrow$ bcc. ${ }^{21,22}$ In the last decade, via synchrotron radiation experiments, it was found that the cubic structure (bcc) transforms to a higherpressure phase with face-centered cubic (fcc) superlattice structure at $100 \mathrm{GPa}$, which further transforms into the fcc 


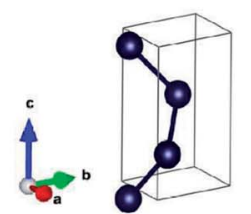

$\mathrm{P} 3,21,0 \mathrm{GPa}$
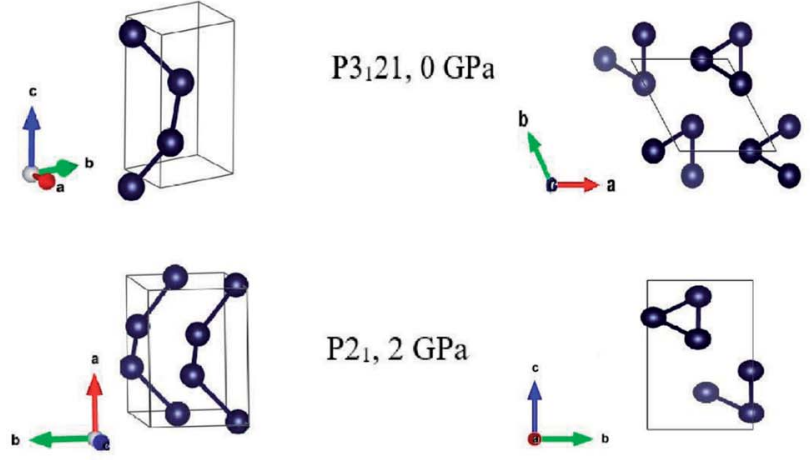

$\mathrm{P} 2{ }_{1}, 2 \mathrm{GPa}$
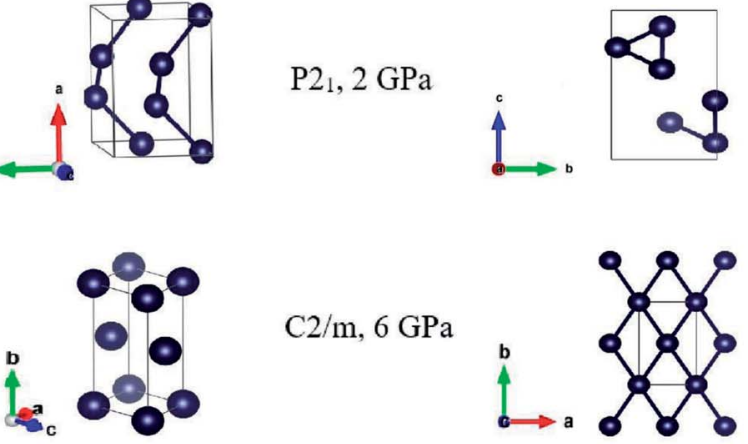

$\mathrm{C} 2 / \mathrm{m}, 6 \mathrm{GPa}$
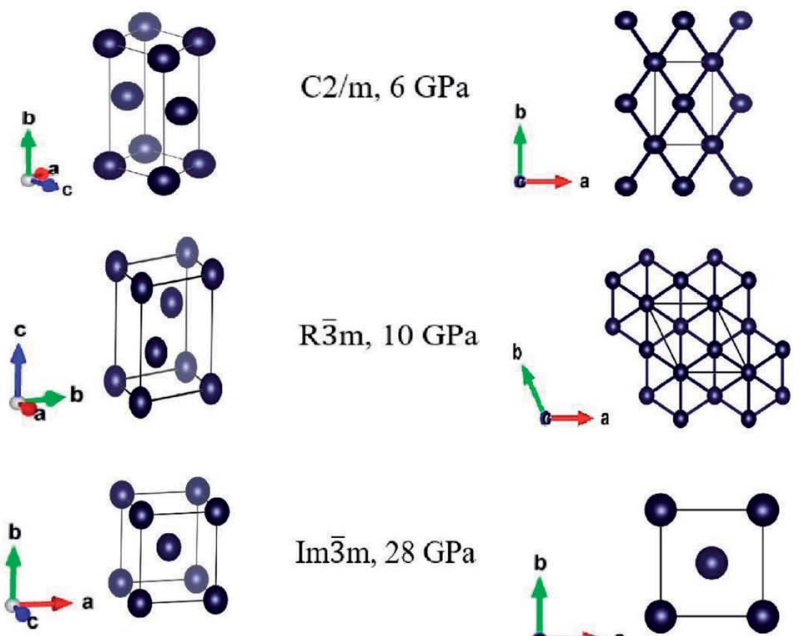

$\operatorname{Im} \overline{3} \mathrm{~m}, 28 \mathrm{GPa}$
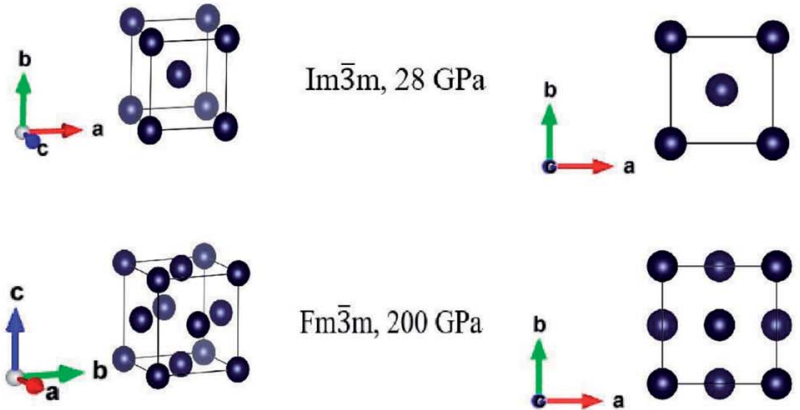

Fig. 13 D-views (left panels) and top-views (right panels) of the optimized structures at the corresponding pressures at which they exhibited lattice stability. For completeness, we show the monoclinic $P 2_{1}$, found at $2 \mathrm{GPa}$, which transformed back into the trigonal $P 3_{1} 21$ after full geometry optimization.

phase at $255 \mathrm{GPa}^{23}$ Clearly, there are still controversies and ambiguities in the question of the crystal structures of tellurium under pressure from the experiments. ${ }^{16,18-22}$

Therefore, it is highly advisable a theoretical and systematic study of the crystal structures of Te by using the state-of-the-art methodologies in crystal structure prediction combined with total energy calculations. ${ }^{24-27}$ In the present work, we employed first-principles crystal structure calculations, by using the particle swarm optimization (PSO) method, as implemented in CALYPSO $^{28}$ code to generate the structures at different pressures and the Vienna Ab initio Simulation Package (VASP) ${ }^{29,30}$ for the total energy optimizations. The structural stability was checked via phonon calculations. ${ }^{31}$ A comparison with the experimentally reported structures was possible via $\mathrm{X}$-ray diffraction patterns calculations.

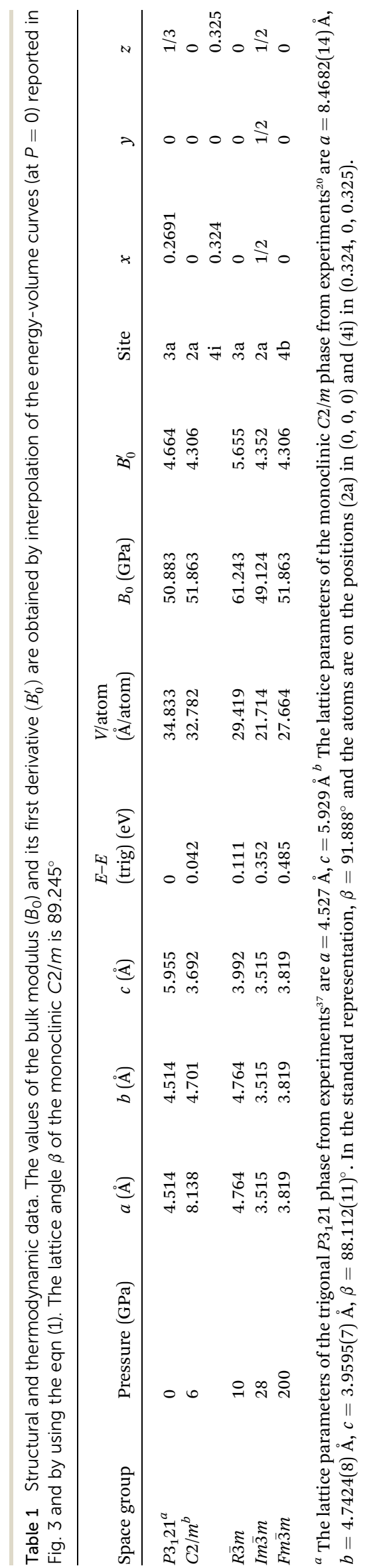




\section{Computational methodology}

The crystal structure analysis based on the particle swarm optimization (as implemented in the CALYPSO package) ${ }^{\mathbf{2 8}}$ was employed to search for possible low-energy structures of tellurium. The entire ensemble of structures were generated by using an initial cell containing 3, 4, 6 atoms and 12 atoms with 30 generations. Each generation contains 20 structures, $60 \%$ of these structures are generated from lowest-enthalpy structure provided by the previous generation, and evolved using particle swarm optimization, while the remaining $40 \%$ will be generated randomly. The pressure scan was run by steps of $2 \mathrm{GPa}$ in the range of $0-32 \mathrm{GPa}$, and by steps of $50 \mathrm{GPa}$ in the range of 100$250 \mathrm{GPa}$. The energy calculations and the geometry optimizations were conducted using VASP ${ }^{29,30}$ with the projector augmented wave (PAW) method..$^{32,33}$ The reliability of the pseudopotential approach has also been confirmed by the fullpotential linearized augmented plane waves approach. The generalized gradient approximation with the Perdew-BurkeErnzerhof (PBE) functional for the exchange correlation was employed..$^{34}$ A plane-wave basis with a cutoff energy of $550 \mathrm{eV}$ was used to expand the wave functions. The Monkhorst-Pack $k$ points mesh was chosen to be no larger than $0.04 \AA^{-1}$. Here, we also adopted the screened hybrid functional HSE06 (ref. 35 and 36 ) to calculate the band gap the $P 3_{1} 21$ phase, using a fixed HF : GGA mixing ratio of $25: 75$ and a screening parameter of $0.2 \AA^{-1}$. We checked the structure stability via phonon calculations, which are performed using $2 \times 2 \times 2$ supercells, as implemented in the Phonopy package. ${ }^{31} \mathrm{X}$-ray powder diffraction (XRD) patterns were simulated by using the Reflex tool implemented in the Materials Studio (MS) package software. The diffraction patterns were calculated over a $2 \theta$-range from $5^{\circ}$ to $45^{\circ}$ with an incremental step size of $0.05^{\circ}$ and for the diffractometer, the silver $(\mathrm{K} \alpha=0.5594075 \AA)$ radiation source, in order to compared our results with the experimental data reported in the literature..$^{20}$

\section{Results and discussion}

Our first-principles crystal structure prediction results confirmed that the ground state is the trigonal $P 3_{1} 21$ phase and that it is stable in the range of 0-6 GPa. In the range 6-10 GPa we found a monoclinic $C 2 / m$ structure, which transformed into the trigonal $R \overline{3} m$ at pressure higher than $10 \mathrm{GPa}$. As increasing further the pressure this trigonal structure in the rhombohedral representation transformed into a body center cubic structure $\operatorname{Im} \overline{3} m$, stable at $28 \mathrm{GPa}$. At very high pressures, from $100 \mathrm{GPa}$ the bcc structure transformed into the face centered cubic $F m \overline{3} m$ phase, stable at $200 \mathrm{GPa}$. The three-dimensional and top views of the corresponding conventional unit cells are displayed in Fig. 1, and the structural and thermodynamic data are reported in Table 1.

The $P 3_{1} 21$ phase has a trigonal crystal structure with helical chains parallel to the crystallographic $c$-direction. As reported, ${ }^{\mathbf{1 6}, 38}$ we also obtained the monoclinic $P 2_{1}$ phase in our crystal structure calculations at $2 \mathrm{GPa}$, but it transformed back into the trigonal structure $P 3_{1} 21$ after full geometry

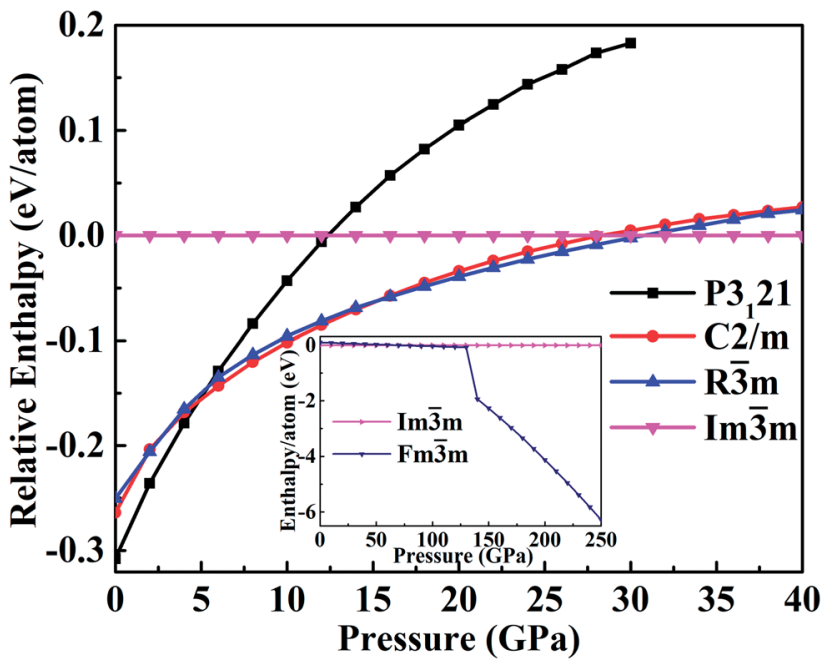

Fig. 2 The calculated enthalpies relative to the high-pressure $1 \mathrm{~m} 3 \mathrm{~m}$ phase as a function of the pressure.

optimization. We find an excellent consistency between the crystal structures from our CALYPSO search ${ }^{28}$ and the previous experiments. $^{20}$ The lattice parameters of the trigonal $P 3_{1} 21$ structures are in good agreement with the experimental values. $^{37}$

The enthalpy as a function of the pressure, reported in Fig. 2, can help to identify the phase transitions. This confirms that the $P 3_{1} 21$ phase is the most stable structure up to $4 \mathrm{GPa}$. As the pressure increases up to $5 \mathrm{GPa}$, the $C 2 / m$ phase has lower enthalpy than that of $P 3_{1} 21$ phase and becomes stable at $6 \mathrm{GPa}$. At pressure less than $15 \mathrm{GPa}$, the monoclinic $C 2 / m$ phase transforms into the trigonal $R \overline{3} m$ phase. Experimentally, this transition was suggested in the pressure range of 10.6$27 \mathrm{GPa} .^{18,20}$ Furthermore, the transformation from the trigonal $R \overline{3} m$ to the cubic $\operatorname{Im} \overline{3} m$ occurs at about $30 \mathrm{GPa}$. The X-raydiffraction experiments ${ }^{20}$ report the transition from the rhombohedral structure to the body-centered-cubic structure at $27 \pm$ $3 \mathrm{GPa}$. The cubic Im $\overline{3} m$ structure then transform into the fcc $F m \overline{3} m$ structure at pressure larger than $100 \mathrm{GPa}$.

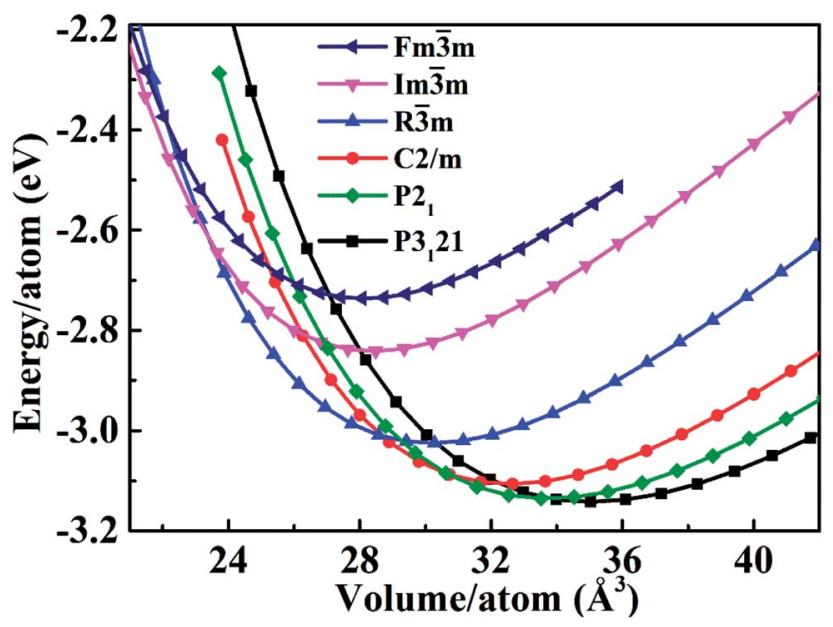

Fig. 3 The total energy as a function of the volume (at $P=0$ ). 

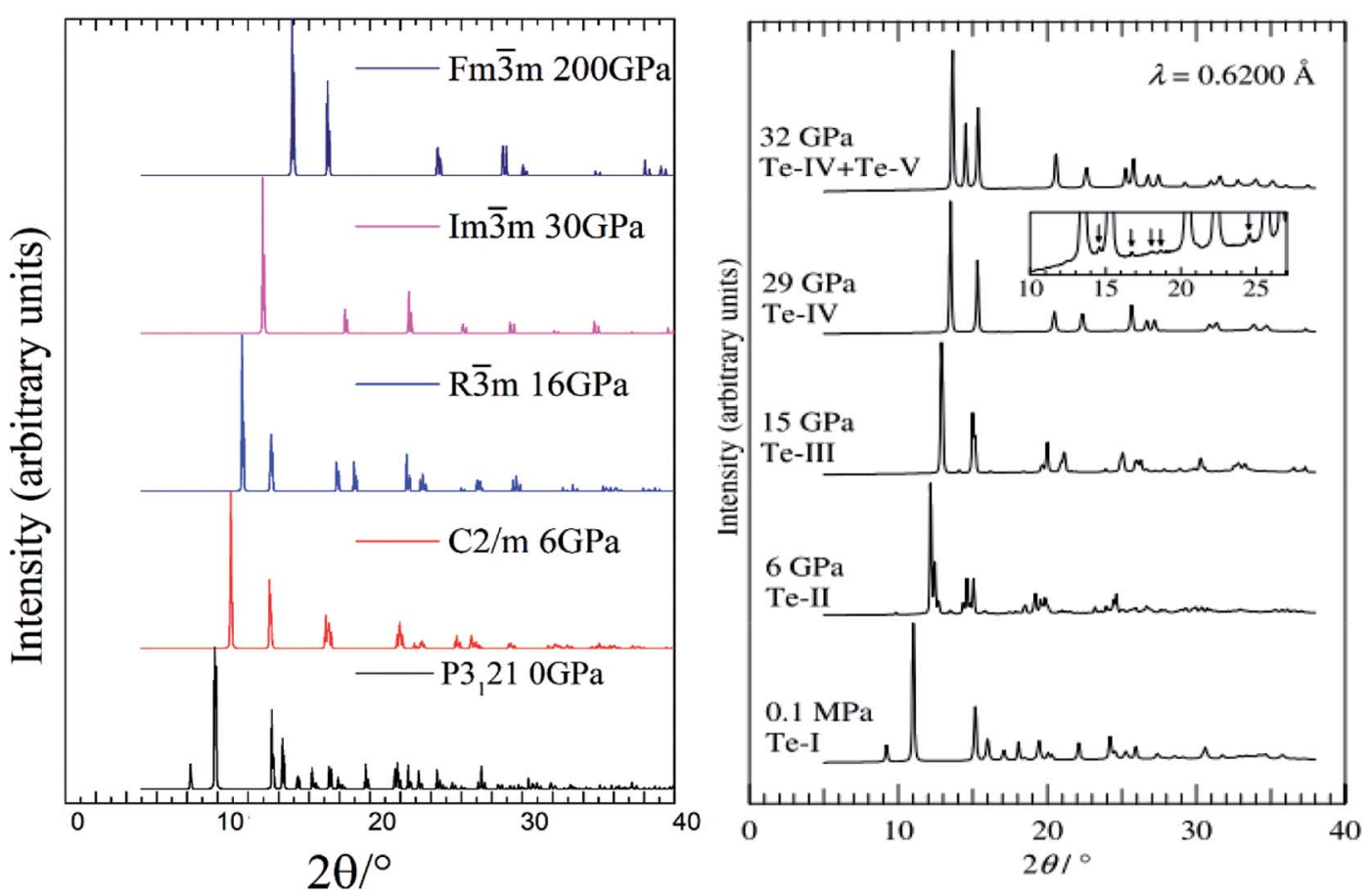

Fig. 4 Simulated XRD patterns of the optimized structures at different pressures (left panel) compared with experiment work (right panel). ${ }^{20}$

We calculated the bulk modulus and its first derivative with respect the volume by fitting, for each phase, the total energy as a function of the volume with a third-order Birch-Murnaghan equation: ${ }^{39}$

$$
\begin{aligned}
E(V)= & E_{0}+\frac{9 V_{0} B_{0}}{16}\left\{\left[\left(\frac{V_{0}}{V}\right)^{2 / 3}-1\right]^{3} B_{0}^{\prime}+\left[\left(\frac{V_{0}}{V}\right)^{2 / 3}-1\right]^{2}[6\right. \\
& \left.\left.-4\left(\frac{V_{0}}{V}\right)^{2 / 3}\right]\right\}
\end{aligned}
$$

where $V_{0}$ is the volume per formula unit at ambient pressure, $V$ is the volume per formula unit at pressure $P$ given in GPa, $B_{0}$ is the isothermal bulk modulus, and $B_{0}^{\prime}$ is the first derivative of the bulk modulus with respect to the pressure. The values of $B_{0}$ and $B_{0}^{\prime}$ are listed in Table 1 . Our energy-volume curves, shown in Fig. 3, are in good agreement with computational works reported in the literature. ${ }^{40}$ From the energy-volume curves we can confirm that the monoclinic structure $C 2 / \mathrm{m}$ represents a phase stable at intermediate pressure between the ground-state, trigonal $P 3_{1} 21$, and the high pressure trigonal $R \overline{3} m$ structure.

Actually, the structural complexity of tellurium under pressure remains elusive even experimentally. ${ }^{20,21,23}$ The very early Xray diffraction study reported a monoclinic to orthorhombic phase transition at $6.6 \mathrm{GPa} .{ }^{16}$ However, such orthorhombic state might be attributed to a different representation of the monoclinic $P 2_{1}$ structure, which we found having the lattice angle $\beta$ very close to $90^{\circ}$, in good agreement with the experimentally reported phase transitions. ${ }^{29}$ Our enthalpy-pressure curves show that the transition sequence is: trigonal $P 3_{1} 21$ to monoclinic $C 2 / m$ (at $6 \mathrm{GPa}$ ) to trigonal $R \overline{3} m$ (at $10 \mathrm{GPa}$ ) to cubic $\operatorname{Im} \overline{3} m$ (at $28 \mathrm{GPa}$ ) and then to the cubic $F m \overline{3} m$ at pressure as large as $200 \mathrm{GPa}$.

Our calculated XRD patterns of the $P 3_{1} 21, C 2 / m, R \overline{3} m, \operatorname{Im} \overline{3} m$ and $F m \overline{3} m$ structures are shown in Fig. 4 for a comparison with experiments. $^{20}$ We have found excellent consistency between the calculated and the experimental XRD patterns of $P 3_{1} 21$ phase. ${ }^{20}$ As the pressure increases, the small peak below $10^{\circ}$ completely disappears in the $C 2 / m$ and $R \overline{3} m$ phases. Very interestingly, if we combine our simulated XRD of $R \overline{3} m$ and $\operatorname{Im} \overline{3} m$ structures at $28 \mathrm{GPa}$, we could perfectly reproduce the phase coexistence, Te-IV $+\mathrm{Te}-\mathrm{V}$, reported at $32 \mathrm{GPa}$ experimentally. ${ }^{20}$

It is well known that the soft phonon modes with negative frequencies are indicative of the dynamical instability of a crystal. To get a better understanding of the dynamical and mechanical stability of these phases, we calculated the phonon dispersions and elastic constants based on the first principles and direct force-constant approaches by using the Phonopy package. ${ }^{31}$ The calculated phonon spectra of different tellurium phases are displayed in Fig. 5. We observe that the interplay between the strong covalent intra-chain and weak inter-chain interactions in trigonal $P 3_{1} 21$ tellurium ${ }^{\mathbf{4 1 , 4 2}}$ gives rise to the phonon band gap between the lower and higher optical phonon branches. This is consistent with the DFT calculation results ${ }^{6}$ and also the experimental dispersion curves reported in the literature. $^{43}$

The absence of negative phonon frequencies or any soft phonon mode, confirms the lattice stability of the five structures. In particular, the monoclinic $C 2 / \mathrm{m}$ structure is stable at $6 \mathrm{GPa}$, the trigonal $R \overline{3} \mathrm{~m}$ structure at $10 \mathrm{GPa}$. The two cubic structures are stable at pressure larger than $28 \mathrm{GPa}$ and 

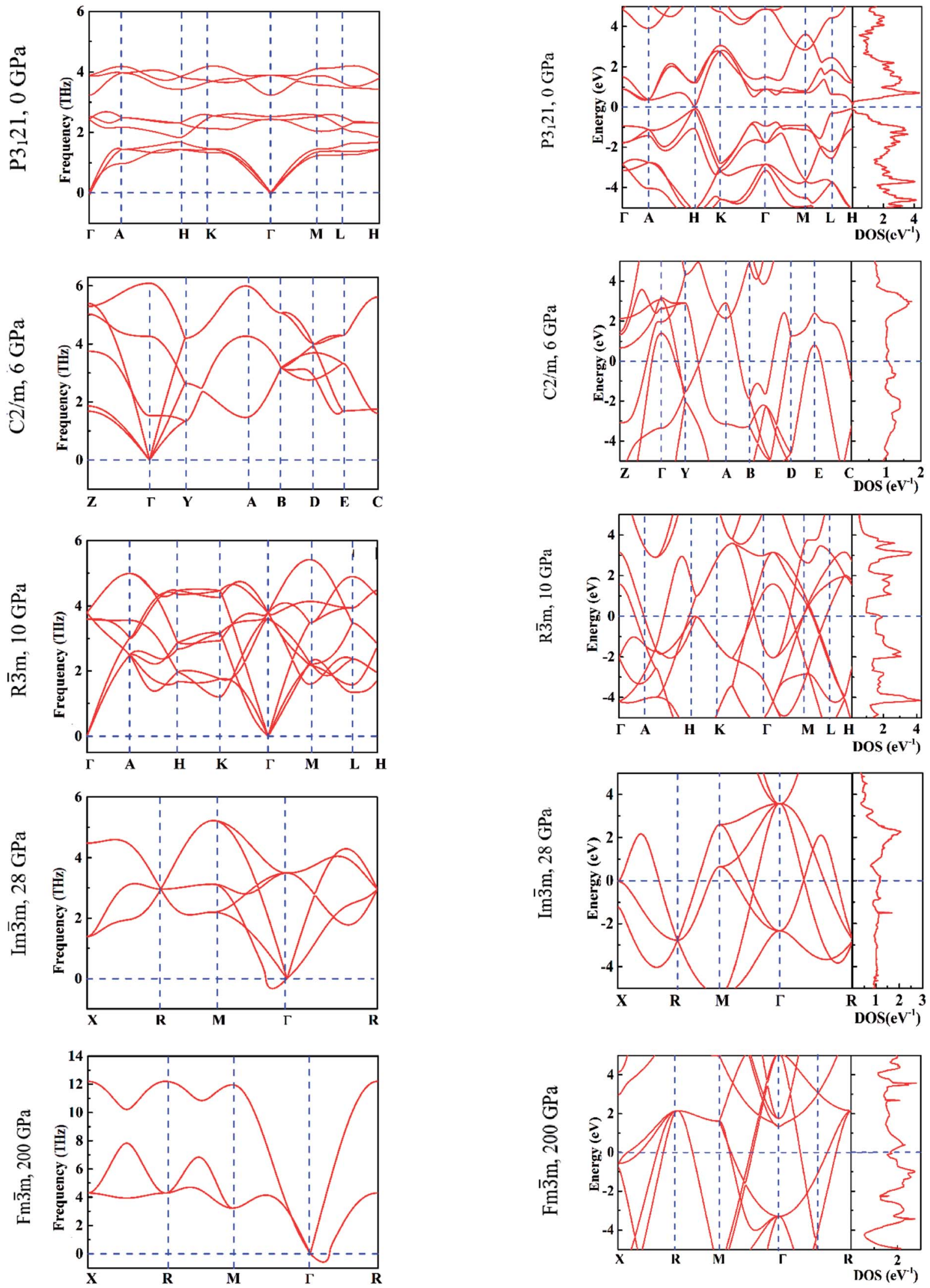

Fig. 5 The calculated phonon dispersion curves under different hydrostatic pressures.

Fig. 6 The calculated electronic band structures (left panels) and density of states (right panels). 
$200 \mathrm{GPa}$, respectively, at which the corresponding transitions occur. The dynamical instabilities of the two cubic structures visible in the corresponding phonon dispersion curves in Fig. 5 are attributable to translational modes at the respective phase transitions.

In addition, we calculated the phonon spectra of the monoclinic $P 2_{1}$ phase at $2 \mathrm{GPa}$ and found that it is similar to the trigonal $P 3_{1} 21$ structure, confirming that it is not a distinct phase.

In Fig. 6 we report the calculated electronic band structures and the electronic density of states (DOS) for $P 3{ }_{1} 21$ at $0 \mathrm{GPa}, C 2 /$ $m$ at $6 \mathrm{GPa}, R \overline{3} m$ at $10 \mathrm{GPa}, \operatorname{Im} \overline{3} m$ at $28 \mathrm{GPa}$ and $F m \overline{3} m$ at $200 \mathrm{GPa}$. We found that the $P 3_{1} 21$ phase has a direct band gap of $0.15 \mathrm{eV}$ by using PBE functional and $0.55 \mathrm{eV}$ by using a hybrid functional. This is in good agreement with the previous ab initio calculations, ${ }^{40}$ and the experimental value of a direct gap of $0.33 \mathrm{eV}^{44}$ The pressure induces the phase transition from semiconductor to metal, as all phases at pressure above zero are metallic. ${ }^{16,18,38}$

\section{Conclusions}

We investigated the allotropes of tellurium crystal under pressure by combining first-principles total energy calculations (VASP) and crystal structure prediction methodology (CALYPSO). The calculated enthalpy-volume curves indicate that tellurium transforms from the ground state, trigonal $P 3_{1} 21$ phase, to the $\alpha$-Po type structure, trigonal space group $R \overline{3} m$, via the monoclinic $C 2 / m$ phase in the range of $0-10 \mathrm{GPa}$. As increasing the pressure, the rhombohedral structure of the $R \overline{3} m$ phase transforms into a stable body centered cubic structure (bcc), space group $\operatorname{Im} \overline{3} m$, at $28 \mathrm{GPa}$. At higher pressure, larger than $100 \mathrm{GPa}$, the bcc structure transforms into a face centered cubic structure, space group $F m \overline{3} m$, whose lattice turns stable at $200 \mathrm{GPa}$. The comparison of the calculated and experimental XRD patterns indicates a good agreement of the sequence of the phases upon the applied pressure. ${ }^{20}$ We calculated the phonon spectra of all phases and found that the monoclinic $C 2 / \mathrm{m}$ is stable at $6 \mathrm{GPa}$, the trigonal $R \overline{3} m$ at $10 \mathrm{GPa}$, the cubic $\operatorname{Im} \overline{3} m$ at $28 \mathrm{GPa}$ and the cubic $F m \overline{3} m$ at $200 \mathrm{GPa}$. In addition, the band structures confirmed the pressure-induced transition from the ground state semiconductor phase to the metallic phases at pressure larger than $2 \mathrm{GPa}$.

\section{Conflicts of interest}

There are no conflicts to declare.

\section{Acknowledgements}

This work was supported by the National Key Basic Research Program of China (Grant No. 2015CB921600), the National Natural Science Foundation of China (Grants No. 51672171 and 51861145315), the fund of the State Key Laboratory of Solidification Processing in NWPU (SKLSP201703), and the supercomputing services from AM-HPC.

\section{References}

1 J. P. Dismukes, L. Ekstrom, E. F. Steigmeier, I. Kudman and D. S. Beers, Thermal and Electrical Properties of Heavily Doped Ge-Si Alloys up to $1300^{\circ} \mathrm{K}$, J. Appl. Phys., 1964, 35(10), 2899-2907.

$2 \mathrm{~W}$. M. Yim and A. Amith, Bi-Sb alloys for magnetothermoelectric and thermomagnetic cooling, Solid-State Electron., 1972, 15, 1141-1161.

3 S. Lin, W. Li, Z. Chen, J. Shen, B. Ge and Y. Pei, Tellurium as a high-performance elemental thermoelectric, Nat. Commun., 2016, 7, 10287.

4 W. Li, Y. X. Wu, S. Q. Lin, Z. W. Chen, J. Li, X. Y. Zhang, L. L. Zheng and Y. Z. Pei, Advances in EnvironmentFriendly SnTe Thermoelectrics, ACS Energy Lett., 2017, 2(10), 2349-2355.

5 M. Wiesner, A. Trzaskowska, B. Mroz, S. Charpentier, S. Wang, Y. Song, F. Lombardi, P. Lucignano, G. Benedek, D. Campi, M. Bernasconi, F. Guinea and A. Tagliacozzo, The electron-phonon interaction at deep $\mathrm{Bi}_{2} \mathrm{Te}_{3}$ semiconductor interfaces from Brillouin light scattering, Sci. Rep., 2017, 7, 16449.

6 H. Peng, N. Kioussis and D. A. Stewart, Anisotropic lattice thermal conductivity in chiral tellurium from first principles, Appl. Phys. Lett., 2015, $107(25), 251904$.

7 H. Peng, N. Kioussis and G. J. Snyder, Elemental tellurium as a chiral p-type thermoelectric material, Phys. Rev. B: Condens. Matter Mater. Phys., 2014, 89(19), 195206.

8 L. Xian, A. Perez Paz, E. Bianco, P. M. Ajayan and A. Rubio, Square selenene and tellurene: novel group VI elemental $2 \mathrm{D}$ materials with nontrivial topological properties, $2 D$ Mater., 2017, 4(4), 041003.

9 Y. Liu, W. Wu and W. A. III Goddard, Tellurium: Fast Electrical and Atomic Transport along the Weak Interaction Direction, J. Am. Chem. Soc., 2018, 140(2), 550553.

10 X.-X. Xue, Y.-X. Feng, L. Liao, Q.-J. Chen, D. Wang, L.-M. Tang and K. Chen, Strain tuning of electronic properties of various dimension elemental tellurium with broken screw symmetry, J. Phys.: Condens. Matter, 2018, 30(12), 125001.

11 S. Gao, Y. Wang, R. Wang and W. Wu, Piezotronic effect in 1D van der Waals solid of elemental tellurium nanobelt for smart adaptive electronics, Semicond. Sci. Technol., 2017, 32(10), 104004.

12 X. Huang, J. Guan, Z. Lin, B. Liu, S. Xing, W. Wang and J. Guo, Epitaxial Growth and Band Structure of Te Film on Graphene, Nano Lett., 2017, 17(8), 4619-4623.

13 K. Nakayama, M. Kuno, K. Yamauchi, S. Souma, K. Sugawara, T. Oguchi, T. Sato and T. Takahashi, Band splitting and Weyl nodes in trigonal tellurium studied by angle-resolved photoemission spectroscopy and density functional theory, Phys. Rev. B, 2017, 95(12), 125204.

14 M. Hirayama, R. Okugawa, S. Ishibashi, S. Murakami and T. Miyake, Weyl Node and Spin Texture in Trigonal 
Tellurium and Selenium, Phys. Rev. Lett., 2015, 114(20), 206401.

15 L. A. Agapito, N. Kioussis, W. A. 3rd Goddard and N. P. Ong, Novel family of chiral-based topological insulators: elemental tellurium under strain, Phys. Rev. Lett., 2013, 110(17), 176401.

16 K. Aoki, O. Shimomura and S. Minomura, Crystal Structure of the High-pressure Phase of Tellurium, J. Phys. Soc. Jpn., 1980, 48(No.2), 551-556.

17 F. P. Bundy and K. J. Dunn, Electrical behavior of Se and Te to pressures of about $500 \mathrm{kbar}$, J. Chem. Phys., 1979, 71(4), 1550-1558.

18 G. Parthasarathy and W. B. Holzapfel, High-pressure structural phase transitions in tellurium, Phys. Rev. B: Condens. Matter Mater. Phys., 1988, 37(14), 8499-8501.

19 Y. Akahama, M. Kobayashi and H. Kawamura, Pressureinduced superconductivity and phase transition in selenium and tellurium, Solid State Commun., 1992, 84, 803-806.

20 M. Takumi, T. Masamitsu and K. Nagata, X-ray structural analysis of the high-pressure phase III of tellurium, $J$. Phys.: Condens. Matter, 2002, 14(44), 10609-10613.

21 C. Hejny and M. I. McMahon, Complex crystal structures of Te-II and Se-III at high pressure, Phys. Rev. B: Condens. Matter Mater. Phys., 2004, 70(18), 184109.

22 C. Hejny and M. I. McMahon, Large structural modulations in incommensurate Te-III and Se-IV, Phys. Rev. Lett., 2003, 91(21), 215502.

23 T. Sugimoto; Y. Akahama; T. Ichikawa; H. Fujihisa; N. Hirao and Y. Ohishi, Bcc-fcc structure transition of Te. in 18th ApsSccm and 24th Airapt, Pts 1-19, ed. W.Buttler, M.Furlanetto and W.Evans. 2014; vol. 500.

24 C. Lu and C. Chen, High-Pressure Evolution of Crystal Bonding Structures and Properties of $\mathrm{FeOOH}$, J. Phys. Chem. Lett., 2018, 9(9), 2181-2185.

25 C. Lu, Q. Li, Y. Ma and C. Chen, Extraordinary Indentation Strain Stiffening Produces Superhard Tungsten Nitrides, Phys. Rev. Lett., 2017, 119(11), 115503.

26 C. Lu, M. Amsler and C. Chen, Unraveling the structure and bonding evolution of the newly discovered iron oxide $\mathrm{FeO}_{2}$, Phys. Rev. B, 2018, 98(5), 054102.

27 C. Zhang, X. Kuang, Y. Jin, C. Lu, D. Zhou, P. Li, G. Bao and A. Hermann, Prediction of Stable Ruthenium Silicides from First-Principles Calculations: Stoichiometries, Crystal Structures, and Physical Properties, ACS Appl. Mater. Interfaces, 2015, 7(48), 26776-26782.

28 Y. Wang, J. Lv, L. Zhu and Y. Ma*, CALYPSO: A method for crystal structure prediction, Comput. Phys. Commun., 2012, 183, 2063-2070.

29 G. Kresse and J. Hafner, Ab initio molecular-dynamics simulation of the liquid-metal-amorphous-semiconductor transition in germanium, Phys. Rev. B: Condens. Matter Mater. Phys., 1994, 49(20), 14251-14269.

30 G. Kresse and J. Hafner, Ab initio molecular dynamics for liquid metals, Phys. Rev. B: Condens. Matter Mater. Phys., 1993, 47(1), 558-561.

31 K. Parlinski, Z. Q. Li and Y. Kawazoe, First-Principles Determination of the Soft Mode in Cubic ZrO2, Phys. Rev. Lett., 1997, 78(21), 4063-4066.

32 G. Kresse and J. Furthmiiller, Efficiency of ab-initio total energy calculations for metals and semiconductors using a plane-wave basis set, Comput. Mater. Sci., 1996, 6(1), 15-50.

33 G. Kresse and J. Furthmüller, Efficient iterative schemes for ab initio total-energy calculations using a plane-wave basis set, Phys. Rev. B: Condens. Matter Mater. Phys., 1996, 54(16), 18.

34 J. P. Perdew, K. Burke and M. Ernzerhof, Generalized Gradient Approximation Made Simple, Phys. Rev. Lett., 1996, 77(18), 3865-3868.

35 A. V. Krukau, O. A. Vydrov, A. F. Izmaylov and G. E. Scuseria, Influence of the exchange screening parameter on the performance of screened hybrid functionals, J. Chem. Phys., 2006, 125(22), 224106.

36 J. Heyd, G. E. Scuseria and M. Ernzerhof, Hybrid functionals based on a screened Coulomb potential, J. Chem. Phys., 2003, 118(18), 8207-8215.

37 S. United and H. E. Swanson, Standard X-ray diffraction powder patterns. U. S. Dept. of Commerce, National Bureau of Standards, Washington, 1953,p volumes.

38 J. C. Jamieson and D. B. Mcwhan, Crystal Structure of Tellurium at High Pressures, J. Chem. Phys., 1965, 43(4), 1149-1152.

39 J.-P. Poirier and A. Tarantola, A logarithmic equation of state, Phys. Earth Planet. Inter., 1998, 109(1-2), 1-8.

40 J. Li, A. Ciani, J. Gayles, D. A. Papaconstantopoulos, N. Kioussis, C. Grein and F. Aqariden, Non-orthogonal tight-binding model for tellurium and selenium, Philos. Mag., 2013, 93(23), 3216-3230.

41 R. M. Martin, G. Lucovsky and K. Helliwell, Intermolecular bonding and lattice dynamics of Se and Te, Phys. Rev. B: Condens. Matter Mater. Phys., 1976, 13(4), 1383-1395.

42 F. Mauri, O. Zakharov, S. d. Gironcoli, S. G. Louie and M. L. Cohen, Phonon Softening and Superconductivity in Tellurium under Pressure, Phys. Rev. Lett., 1996, 77(6), 1151-1154.

43 B. M. Powell and . Marte, The lattice dynamics of tellurium, J. Phys. Chem. Solids, 1975, 36, 1287-1298.

44 V. B. Anzin, M. I. Eremets, Y. V. Kosichkin, A. I. Nadezhdinskii and A. M. Shirokov, Energy Gap in Tellurium under Pressure, Phys. Status Solidi, 1977, 42, 385-390. 